\title{
Isolated palmar dislocation of the fifth carpometacarpal joint: a case report of rare injury
}

\begin{abstract}
Introduction: Isolated palmar dislocation of fifth carpometacarpal joint is a very rare injury, occurs commonly direct blow, fall and RTA. It is missed to diagnosis if not carefully evaluate and in poly trauma patients. Careful examination and radiographic evaluation is paramount of diagnosis the injury. The goal of treatment is early reduction and stabilize the joint. All functions with normal grip strength regain within 6 months. The objective of this case report to describe the management of an isolated palmar dislocation of fifth carpometacarpal joint.
\end{abstract}

Case report: A 35 years male admitted with severe pain and swelling of his right hand after blow to a hand object. Clinically the little finger was abducted and extended. Also shortening of fifth ray and depress in proximal right carpus ondorsomedial aspect. AnteroPosterior and lateral radiographs of right hand and wrist evaluate an isolated palmar dislocations of fifth carpometacarpal joint. Initially hand evaluation and NSAID started, then on the next day of injury closed reduction and percutaneous k-wire fixation done under General Anaesthesia. Reduction was stable and the base of fifth metacarpal bone in position confirmed by postoperative radiographs. He regain his normal hand function after 3 months of reduction.

Conclusion: Isolated fifth carpometacarpal joint dislocation likely to be missed.Delayed diagnosis many cause of chronic residual pain and unstabilized the reduction. After reduction and stabilization close follow up and radiological evaluation needed for successful good outcome the treatment of the injury.

Keywords: fifth carpometacarpal (CMC) joint, palmar dislocation, active and passive movement, percutaneous.

Message of the case report: Isolated palmar dislocation of fifth carpometacarpal joint diagnosis confirmed by careful examination and proper radiological evaluation. Successfully manage by closed reduction and stabilize the joint with k-wire. Also close follow-up with regular interval, encourage hand and finger movements satisfactory functional regain of the hand within very minimal short period of 3-6 months.
Volume II Issue 2 - 2019

\author{
Chowdhury Foyzur Rob,' Md. Jahangir \\ Hossain, ${ }^{2}$ Md. Ekhlasur Rahman, ${ }^{3}$ Md. Abdul \\ Awal, ${ }^{4}$ Tanvir Ahmed ${ }^{5}$ \\ 'Senior Consultant, Department of Orthopaedics, Al-Haramain \\ Hospital, Bangladesh \\ ${ }^{2}$ Senior Registrar, Department of Orthopaedics, Al-Haramain \\ Hospital, Bangladesh \\ ${ }^{3}$ Student of MS in Orthopaedics Course, Sylhet MAG Osmani \\ Medical College, Bangladesh \\ ${ }^{4}$ Registrar, North East Medical College, Bangladesh \\ ${ }^{5}$ Medical Officer, North East Medical College, Bangladesh
}

Correspondence: Chowdhury Foyzur, Rob Senior Consultant, Department of Orthopaedics, Al-Haramain Hospital, Kazi Tower, SubhaniGhat, Sylhet-3100, Bangladesh, Tel +880 I7I5250800, Email foyzurrob@gmail.com

Received: February 27, 2019 | Published: April 10,2019

\section{Introduction}

Isolated fifth carpometacarpal joint dislocation is a very rare injury $^{1}$ but among the carpometacarpal joint dislocation, fifth carpometacarpal joint most commonly affected than others. Usually displace dorsally. Carpometacarpal joint dislocation usually occurs in polytrauma patients and associated with fracture of metacarpal bones. The dislocation can be missed to diagnosis, so careful examination and attention should be need. H/O - Fall, blow or RTA are predisposing causes. Young male persons are more affected. Average age 30 years of 12 reported cases. Right hand are commonly involved. ${ }^{1-8}$

Patients are present with pain and swelling of the affected hand. Diagnosis confirmed by radiographs of hand with wrist. Treatment of fifth carpometacarpal joint dislocation are closed or open reduction and k-wire fixation required if joint unstable. The goal of treatment is early reduction. ${ }^{8}$ Delayed reduction of carpometacarpal joint dislocation may cause of chronic residual pain. Here we report a case of isolated palmar dislocation of fifth carpometacarpal joint of right hand after blow to a hard object, successfully managed by closed reduction and percutaneous $\mathrm{k}$-wire fixation. The man in good scene give written informed consent for print and electronic publication of this case report.

\section{Case report}

A 35 years young man admitted into orthopaedics department of our hospital with severe pain and swelling of right hand after blow to a hard object. He was unable to move right little finger and also painful movement of other fingers. Clinically there was a depression in the dorsomedial aspect of right carpus proximally and swelling rest of the hand. The little finger was abducted and extension in position. Some shortening of fifth digital ray. There was no sign of neurovascular injury. Radiographs of right hand with wrist joint was done. Antero-posterior and lateral view of x-ray, the base of fifth metacarpal was displaced proximally. Phalanges of little finger abducted in antero-posterior view. There was also palmar displacement of fifth metacarpal base in lateral view. There was no other displacement or fracture of carpals, metacarpals and phalanges of right hand. So, isolated palmar dislocation of fifth carpometacarpal joint (right) confirmed by radiographs.

Initially treatment started with NSAID and hand elevation. On the next day hand swelling was minimally subsided, closed reduction and percutaneous k-wire fixation was done under general anaesthesia with c-arm guidance. The reduced base of fifth metacarpal bone fixed to fourth and partly third metacarpal base by passing a k-wire transiously 
which maintain the inter metacarpal joint. Another k-wire passing obliquely through base of fifth metacarpal to hamate, it give stability of hamato-metacarpal joint.

Per-operative radiographs ensure the base of fifth metacarpal placed in position. Clinically there was no depression on dorsomedial aspect of proximal position of Right carpus and little finger deformity was corrected. All finger joints moves freely. Proper dressing of k-wire entering points done. Finally apply cock-up slab for immobilization of wrist joint and metacarpal bones. Post-operative $\mathrm{x}$-ray ensure the base of fifth metacarpal and both k-wires in position.

After 1 week the cock-up slab was removed during first follow up. Swelling of Right hand fully subside. Blood circulation intact. Nerve sensation and fingers movements are normal. Encourage finger movements. After 3 weeks both k-wires removed on second follow up. The stability of fifth carpometacarpal joint checked both clinically and radiologically. Also the Indian salutation test was negative. Slight tenderness at fifth carpometacarpal joint with moderate stretch Encourage both active and passive movement of fingers and wrist joints. After 6 weeks during third follow up, slight tenderness on fifth carpometacarpal joint with deep stretch. All movements of wrist and hand were free. He started motor bike ride. After 3 months, on fourth follow up, the patient regain full functions of injured hand (Right). The grip strength was fully satisfactory (Figure 1-5).

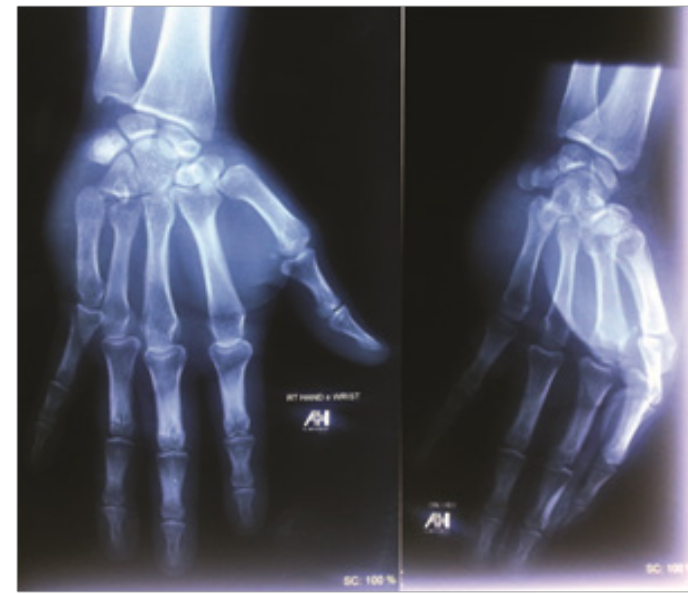

Figure I Preoperarive Radiographic view of AP \& Oblique.

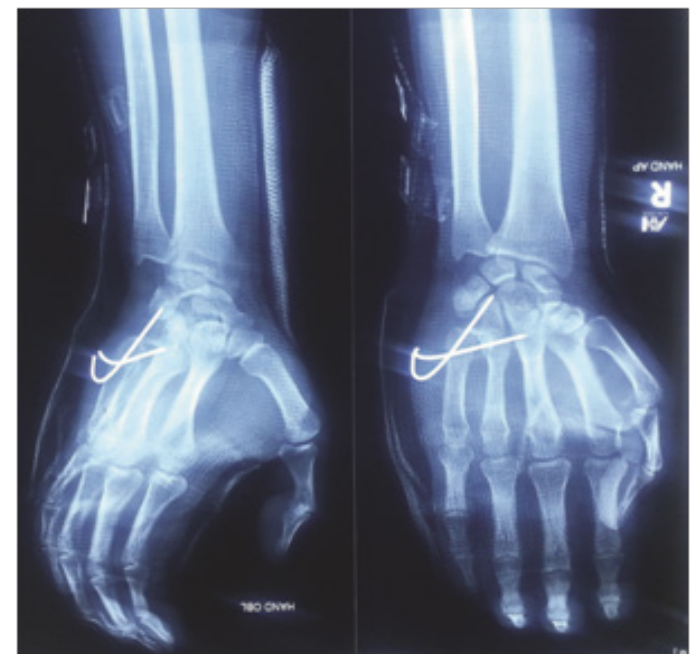

Figure 2 Postoperative Radiographic view of AP and Oblique.

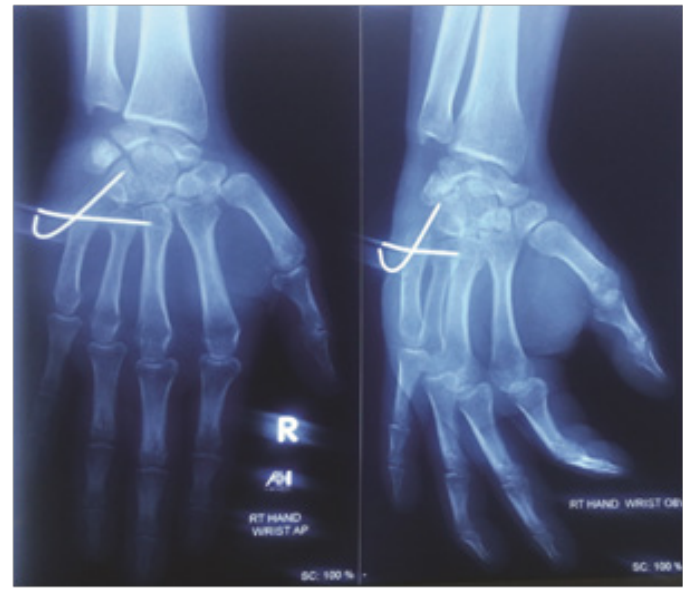

Figure 3 Post operative follow up after one week (Removal of cast and allow movement of the hand) Radiographic view AP \& Oblique.

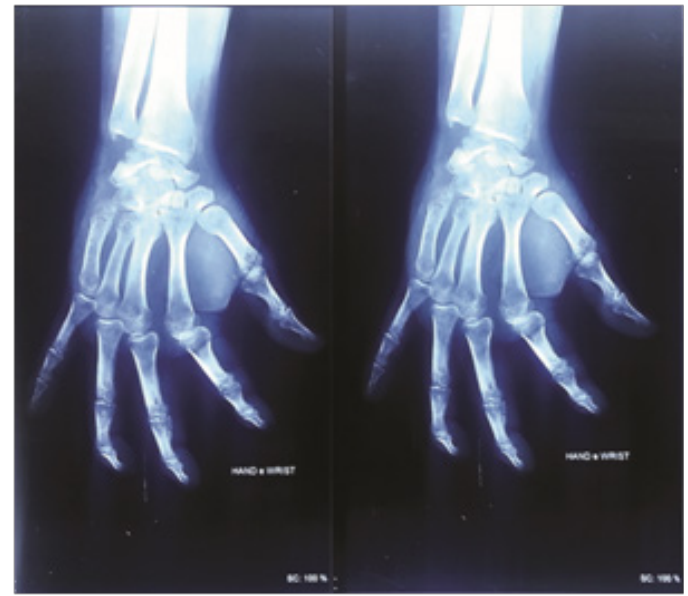

Figure 4 Postoperative follow up after 3 weeks, removal of k-wire. Radiographic view AP \& Oblique after k-wire removal.
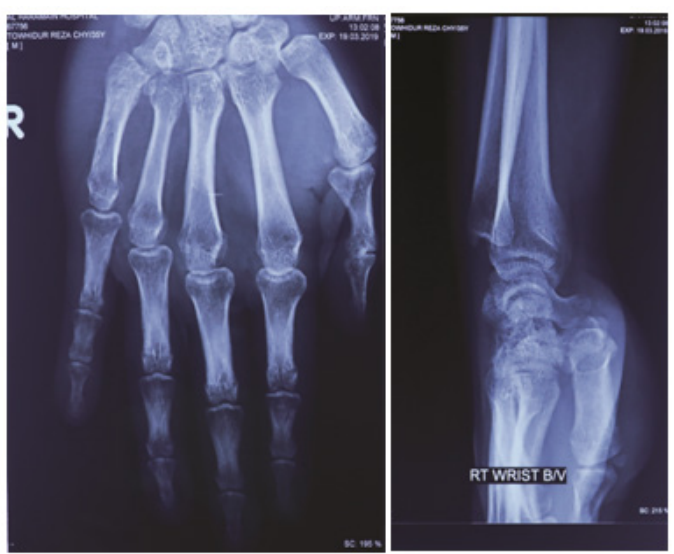

Figure 5 Follow up radiograph after 3 months of reduction.

\section{Discussion}

Carpometacarpal joint dislocation are uncommon injury and less than $1 \%$ of total hand and wrist injuries ${ }^{2,4}$ carpometacarpal joint dislocation most commonly affected the fifth carpometacarpal joint, ${ }^{7}$ associated with hamate fracture or fracture base of the fifth metacarpal or with another carpometacarpal joint dislocation are 
more frequent than isolated fifth carpometacarpal joint dislocation. ${ }^{3}$ Isolated dislocation of fifth carpometacarpal joint is a very rare and high velocity injury. ${ }^{1,4} \mathrm{McWorter}$ first reported and discus isolated fifth carpometacarpal joint dislocation in 1918.,

The injury is due to direct blow transmitted to the dorsoulnar aspect of the fifth metacarpal base, with $\mathrm{H} / \mathrm{O}-$ blow, fall, assault, RTA resulting in disruption of the supporting peri-articular soft tissue. ${ }^{6}$ The hamatometacarpal articulation (fifth carpometacarpal joint) is a saddle joint, with a convex base of fifth metacarpal fitting into a concave facet of the hamate. Both bones have an additional flat facet for articulation with fourth metacarpal bone. This special feature of fifth carpometacarpal joint, differentiate it from other carpometacarpal joints. It's radioulnar stant in the frontal plane allow $10-20^{\circ}$ of supination, when opposing the little finger to the thumb. This additional mobility decreased stability of joint with increased incidence of fifth carpometacarpal joint dislocation, compared with fourth carpometacarpal joint. ${ }^{6}$ Stability of the fifth carpometacarpal joint provided by pisometacarpal ligament, dorsal and palmar carpometacarpal ligaments and intermetacarpal ligaments. The intermetacarpal ligaments constitutes the primary restraint. Also the Extensor Carpi Ulnaris (ECU) and the Flexion Carpi Ulnaris (FCU) tendons insert into the base of fifth metacarpal bone. ${ }^{1,3,4}$

Carpometacarpal joint dislocation are classified as dorsal and palmar (volar) dislocations, dorsal dislocation are more frequent than palmar dislocation, ${ }^{2-4}$ due to thick and strong palmar ligamentous complex. ${ }^{4,5}$ Divergent simultaneous carpometacarpal joint dislocation involving differents digits is another rare type of injury. ${ }^{1,3,4}$

In 1965 Nalebuff classified isolated palmar dislocation of fifth carpometacarpal joint into radiopalmar and ulnopalmar groups according to the direction of displacement of the base of fifth metacarpal. ${ }^{2,3}$ Severity of displacement depends on position of hand, wrist and intensity of force applied. In radiopalmar dislocation of fifth carpometacarpal joint all ligament and tendon attachments are tear and attachments are intact in ulnopalmar dislocation. ${ }^{2,3}$ The deep motor branch of the ulnar nerve lies palmar aspect of the fifth carpometacarpal joint, as it's courses around the hook of the hamate and is vulnerable to injury in palmar dislocation also in dorsal dislocation. A careful neurological assessment of the injured hand is essential. ${ }^{1-3,6}$

We examine the affected right hand of our patient for neurovascular assessment. There was intact both sensory and motor function of ulnar, median and radial nerves supply to hand. Blood circulation was intact and sufficient, as rapid capillary refill of nail beds, $\mathrm{SpO}_{2}-$ $99 \%$ and no sign of ischemia, as no skin discoloration.

Carpometacarpal joint dislocation can be easily missed and failure to diagnosis may be due to pain and weakness of grip, usually in polytrauma patients and also overlapping bony shadow of hand radiographs. Delayed diagnosis of fifth carpometacarpal joint dislocation lead to disruption of both transverse and longitudinal arches of hand, ${ }^{6}$ result in poor functional outcome and chronic residual pain. Careful examination and radiographic evaluation is paramount of diagnosis the injury. $2,4,6$

Isolated fifth carpometacarpal joint dislocation is common in young adult male, around 20-40 years of age and most of the cases right hand was injured. ${ }^{1-8}$ High velocity trauma i.e- RTA are main cause. However low intensity traumas like- fall from height and punches a clenched fist striking to a hard surface on its ulnar side may cause this injury. ${ }^{6}$ There is pain and swelling at the base of fifth metacarpal also dorsum of hand. Axial deformity and rotation of little finger with apparent shortening of fifth ray. There is also depression at base of fifth metacarpal at dorsomedial aspect in palmar dislocation. Tenderness on stretch at the base of metacarpal. ${ }^{1-4}$

In our case, the patient was a 35 years young adult male, $\mathrm{H} / \mathrm{O}$ - direct blow to a hard object, by his right hand. He was presented with severe pain and swelling dorsum of right hand. On the dorsomedial aspect, there was a depression at the base of fifth metacarpal bone. The little finger was abducted and extended with little shortening of fifth ray. Antero-posterior or Posterior-anterior, oblique and true lateral views of injured hand should be obtain for radiological evaluation. ${ }^{6}$ A lateral view in $30^{\circ}$ pronation of the hand provide and improved view of fifth carpometacarpal joint. In the case we reported, there was isolated palmar dislocation of right fifth carpometacarpal joint diagnosed by both antero-posterior and lateral view of $30^{\circ}$ pronated position of hand, as the base of fifth metacarpal bone displaced both proximally and anterior to hamate. Also there was no others involvement of fracture and dislocation.

Treatment of the isolated fifth carpometacarpal joint dislocation still controversial. Close reduction with cast immobilization and closed reduction with percutaneous k-wire or open reduction with internal fixation are advocated. ${ }^{8}$ Immediate reduction and immobilization or k-wire fixation is the ultimate goal of the treatment of the injury. Early diagnosis is the key of success. The injury easily reduced by manipulation, apply longitudinal traction and direct pressure on metacarpal base, this may be successful up to 10 days after the injury. There after unstable reduction should be fixed by k-wire. After 3 weeks, open reduction may be required. ${ }^{6}$ Closed reduction usually done under Local Anaesthesia or Sedation, but k-wire fixation and open reduction require regional block or General Anaesthesia.

Closed reduction of the dislocated fifth carpometacarpal joint with cast immobilization is an option, however due to the degree of soft tissue disruption the injured joint is often unstable. Percutaneous k-wire fixation is required to restore joint stability and facilitate soft tissue healing. ${ }^{7}$ Radiopalmar dislocation are inherently unstable because of the tearing of all ligament and tendon attachments on the base of fifth metacarpal bone. However, in case of closed reduction, there is a higher risk of redislocation of carpometacapal joint. So, closed reduction and k-wire fixation is the recommended treatment for carpometacarpal joint dislocation.

In our case, over night hand elevation swelling of hand minimally subside, then on the next day closed reduction and percutaneous k-wire fixation done under General Anaesthesia, also apply cock-up slab for immobilization of wrist and metacarpals, which facilitate soft tissue healing. Open reduction and k-wire fixation may be required when these is soft tissue interposition which prevent closed reduction of the dislocated joint or in cases of delayed diagnosis. ${ }^{7}$ Also in fracture dislocation need open reduction and internal fixation. ${ }^{4}$

There is no reported case of complications in open reduction and $\mathrm{k}$-wire fixation. But closed reduction failed and redislocation or partial dislocation after removal of cast. ${ }^{1}$ In fracture dislocation cases there was reported complications of palmar interosseous muscle weakness. Loss of sensation due to nerve injury, non-union of metacarpal neck fracture, carpal boss deformity. ${ }^{4}$ Close follow up and serial of radiographic evaluation to ensure maintenance of reduction and recover the hand functions. Cast or k-wires are removed usually at 4-6 weeks. All routine activities with normal grip strength and full range 
of motion at wrist without pain is regain within 6 months, in isolated fifth carpometacarpal joint dislocation. ${ }^{3,4}$ But carpometacarpal joint dislocation of thumb regain full function at 3 years $^{5}$ and $1 \frac{1}{2}$ to 2 years in multiple fracture dislocations. ${ }^{4}$ The man who is reported by us, he regain pain free full functions of hand at 3 months.

\section{Clinical message}

Isolated dislocation of fifth carpometacarpal joint is very rare injury usually missed when associated with polytrauma or other injuries, delaying diagnosis and treatment, result failed closed reduction and poor functional outcome.

\section{Conclusion}

Isolated dislocation of fifth carpometacarpal joint is uncommon injury. Early diagnosis by careful examination and radiological analysis follow to appropriate treatment with regular follow up and radiological evaluation lead to satisfactory outcome. We report such a case, which was successfully managed by closed reduction and percutaneous k-wire fixation.

\section{Acknowledgment}

We declare no fund received for this article from outside of us.

\section{Authors contributions}

C. F. Rob is a major contributor in writing and editing the manuscript, J. Hossain is contribute in writing the manuscript, also analyzed and interpreted the particulars data regarding the injury. E.
Rahman, A. Awal and T. Ahmed had been involved in patients data selection and drafting the manuscript.

\section{References}

1. Anjum R, Roy A, Farooque K, et al. An Isolated Pure Dislocation of Fifth Carpometacarpal Joint:Case Report and Review of Literature. J Orthop Case Rep. 2017;7(2):14-16.

2. Hegde AS, Shenoy RM, Arif S, et al. Isolated Radiopalmar Dislocation of Fifth Carpometacarpal Joint: A Rare Presentation. Am J Orthop (Belle Mead NJ). 2015:44(9):E347-49.

3. Khalid Ibn El Kadi, Mohcine S, Badr A, et al. Isolated radial volar dislocation of the fifth carpometacarpal joint:a rare injury. Pan Afr Med J. 2013;16:90.

4. Pundkare GT, Patil AM. Carpometacarpal Joint Fracture Dislocation of Second to Fifth Finger. Clin Orthop Surg. 2015;7(4):430-435.

5. Elias F, Theodoros S, Christos L, et al. Isolated thumb carpometacarpal joint dislocation:a case report and review of the literature. J Orthop Surg Res. 2010;5:16.

6. Naoufal E, Youssef J, Ayoub B, et al. Pure Isolated Dorsal Hamatometacarpal Dislocation in a Rider: A Case Report and Review of Literature. J Orthop Case Rep. 2018;8(5):29-31.

7. David J Milligan, Neville W Thompson, DiarmaidO' Longain. Volar dislocation of the fifth carpometacarpal joint. Ulster Med J. 2018;87(2):133-134.

8. Maurizio V, Giorgio S, Vincenzo A. Abstract of Pure isolated dorsal dislocation of the fifth carpometacarpal joint. Musculoskeletal Surgery. 2009;93(2):97-100. 